\title{
Laser therapy for onychomycosis in patients with diabetes at risk for foot complications: study protocol for a randomized, double-blind, controlled trial (LASER-1)
}

Leonie Nijenhuis-Rosien ${ }^{1,2^{*}}$, Nanne Kleefstra ${ }^{1,3,4}$, Maurice J Wolfhagen ${ }^{5}$, Klaas H Groenier ${ }^{1,6}$, Henk JG Bilo ${ }^{1,3,6}$ and Gijs WD Landman ${ }^{1,7}$

\begin{abstract}
Background: In a sham-controlled double-blind trial, we aim to establish the efficacy and safety of the local application of laser therapy in patients with diabetes, onychomycosis and risk factors for diabetes-related foot complications. Onychomycosis leads to thickened and distorted nails, which in turn lead to increased local pressure. The combination of onychomycosis and neuropathy or peripheral arterial disease (PAD) increases the risk of developing diabetes-related foot complications. Usual care for high-risk patients with diabetes and onychomycosis is completely symptomatic with frequent shaving and clipping of the nails. No effective curative local therapies exist, and systemic agents are often withheld due to concerns for side effects and interactions.

Methods/Design: The primary aim is to evaluate the efficacy of four sessions of Nd:YAG 1064 nM laser application on the one-year clinical and microbiological cure rate in a randomized, double-blind, sham-controlled design with blinded outcome assessment. Mandatory inclusion criteria are diagnosis of diabetes, risk factors for developing foot ulcers defined as a modified Simm's classification score 1 or 2 and either neuropathy or PAD. A total of 64 patients are randomized to intervention or sham treatment performed by a podiatrist.
\end{abstract}

Discussion: This study will be the first double-blind study that investigates the effects of local laser therapy on onychomycosis, specifically performed in patients with diabetes with additional risk factors for foot complications.

Trial registration: Clinical trials.gov as NCT01996995, first received 22 November 2013.

Keywords: Onychomycosis, Nd:YAG laser, Diabetic foot, Diabetic foot ulcer

\section{Background}

The prevalence of onychomycosis is 2.5 to 2.8 times higher in patients with diabetes [1-3]. The combination of onychomycosis with other risk factors, like neuropathy, increases the risk of developing diabetes-related foot complications [4]. Onychomycosis leads to thickening and sharpening of the nails. Thickening of the nails potentially increases subungual pressure and compromises vascular flow $[5,6]$. Sharpening of the nails can

\footnotetext{
* Correspondence: I.nijenhuis@isala.nl

'Diabetes Centre, Isala, Dr Spanjaardweg 11, 8025 BT Zwolle, the Netherlands ${ }^{2}$ Innofeet Voetencentrum Nijenhuis Podiatry, Simon Stevinweg 13, 8013 NA Zwolle, the Netherlands

Full list of author information is available at the end of the article
}

lead to small skin injuries, which are potential portals of entry for pathogens. In the presence of neuropathy and/ or peripheral arterial disease (PAD), onychomycosis is an additive risk factor for diabetes-related foot complications like foot ulcers, cellulitis, osteomyelitis and gangrene [7-10]. Worldwide, foot ulcers in patients with diabetes are the leading cause of hospitalizations and amputations [11-15]. The development of foot ulcers has been associated with considerable disability [16-18], and the presence of onychomycosis has been associated with various emotional and social problems [19-23].

Treating onychomycosis is increasingly recognized as a potential strategy for preventing diabetes-related foot complications [5,24-26]. As part of the national Dutch 
foot care program, usual care for patients with onychomycosis is symptomatic; every six weeks, nails are skived to normal proportions and sharp edges are clipped and removed. This podiatric treatment aims to prevent ulcer formation and the development of infections through reducing subungual pressure and sharpen nails. Although effective systemic antifungal agents are available, they are often withheld due to concerns of side effects and interactions [27]. Furthermore, studies that investigated antifungal agents mostly excluded patients with diabetes. Despite many claims of efficacy, no proven effective local therapies exist. An effective and safe curative treatment option for onychomycosis could lead to a decrease in diabetes-related foot complications, especially in patients with other risk factors. By reducing the frequency of 'skiving and clipping' treatment sessions and by reducing the development of ulcers and infections caused by treatment-resistant onychomycosis, local therapies could become valuable preventive treatment option [28].

Local laser therapy is becoming increasingly popular as a treatment modality for a variety of dermatologic conditions and is being applied in the treatment of onychomycosis [29]. The use of lasers for improving toenail appearance has become one of the most rapidly approved therapies by the US Food and Drug Administration (FDA) [29]. Unlike pharmacologic agents, laser systems are presumed to have predictable adverse effects [29]. There are few nonrandomized trials and very few randomized studies investigating effect of laser therapy on onychomycosis. None had a double-blind design, and none were performed in patients with diabetes who also had additional risk factors for foot ulcers [29].

The primary aim of this trial is to evaluate the efficacy of four sessions of N-YAG 1064nM laser application on the one-year complete cure rate of onychomycosis in patients with diabetes and risk factors for developing diabetes related foot complications. Secondary outcomes are microbiologic cure rate and improvement in healthrelated quality of life (HRQOL).

\section{Methods/Design}

Primary and secondary care patients with type 1 and type 2 diabetes mellitus with risk factors for developing diabetic foot ulcers and a clinical suspicion of onychomycosis will be recruited from a single-center podiatry practice. Patients will be included after written informed consent and microbiologic confirmation of onychomycosis.

Nail dust is collected from the target nail for microbiologic confirmation with blankophor microscopy, culture and polymerase chain reaction (PCR). PCR is regarded as the gold standard. The PCR is a real-time multiplex PCR based on amplification of ribosomal internal transcribed spacer regions and identification by probes specific for Trichophyton mentagrophytes species complex,
Trichophyton tonsurans, Trichophyton violaceum, Trichophyton rubrum species complex, Microsporum canis, Microsporum audouinii and Epidermophyton floccosum as described by Arabatzis et al. [30].

The target nail is the nail with the highest onychomycosis severity index. The severity of onychomycosis is evaluated with the onychomycosis severity index (OSI) and standardized photographs are made [31]. Evaluation of treatment results will be done by an independent expert panel blinded for treatment allocation. The three experts are experienced health care providers who have experience in the treatment of patients with diabetic foot problems. Health-related quality of life (HRQOl) is evaluated at baseline and after follow-up with a generic questionnaire, the WHO-5, and a disease specific questionnaire the NailQOl [23]. The trial has received research ethics board approval from METC Isala Zwolle (NL46084.075.13/METC nr 13.0885).

\section{Study endpoints}

The primary endpoint is complete cure rate of the target nail. Complete cure is defined as a completely normal nail, or negative microbiological results in case minor abnormalities are present. A minor abnormality is defined as an irregularity of the nails with less than $5 \%$ of the surface area of the target nail at less than $1 / 4$ of the distance of the distal nail edge without hyperkeratosis. Secondary endpoints are microbiologic cure rate of the target nail, complete clinical cure of all affected toes, complete clinical cure of the target nail, markedly clinically improvement of the target nail, onychomycosis severity index below 6 (in patients with scores $>6$ at study entry) of the target nail, changes in the affected surface of the target nail, changes in affected surface of all nails with a clinical diagnose of infection, free of subungual hyperkeratosis and changes in HRQOL. A 'markedly clinical nail improvement' is defined as a nail with less than $10 \%$ abnormalities and without hyperkeratosis after 52 weeks.

\section{Patient selection criteria}

Patients with clinical suspicion and microbiologic confirmation of onychomycosis are randomized to laser treatment or sham, see Figure 1.

Mandatory inclusion criteria are diagnosis of T1DM or T2DM, 18 years or older, at risk for diabetic foot ulcers defined by a modified Simm's classification score 1 or 2 with either neuropathy or PAD [32], and nail involvement of at least $25 \%$ of the target nail [3]. See Table 1 for the modified Simm's classification.

Exclusion criteria are no microbiologic confirmation, Simms' classification score 3 , the presence or history of diabetic foot ulcers, ischemic pain, ankle brachial index $<0.9$, patients receiving dialysis, severe renal insufficiently (eGFR below $30 \mathrm{ml} / \mathrm{min}$ ), a documented 


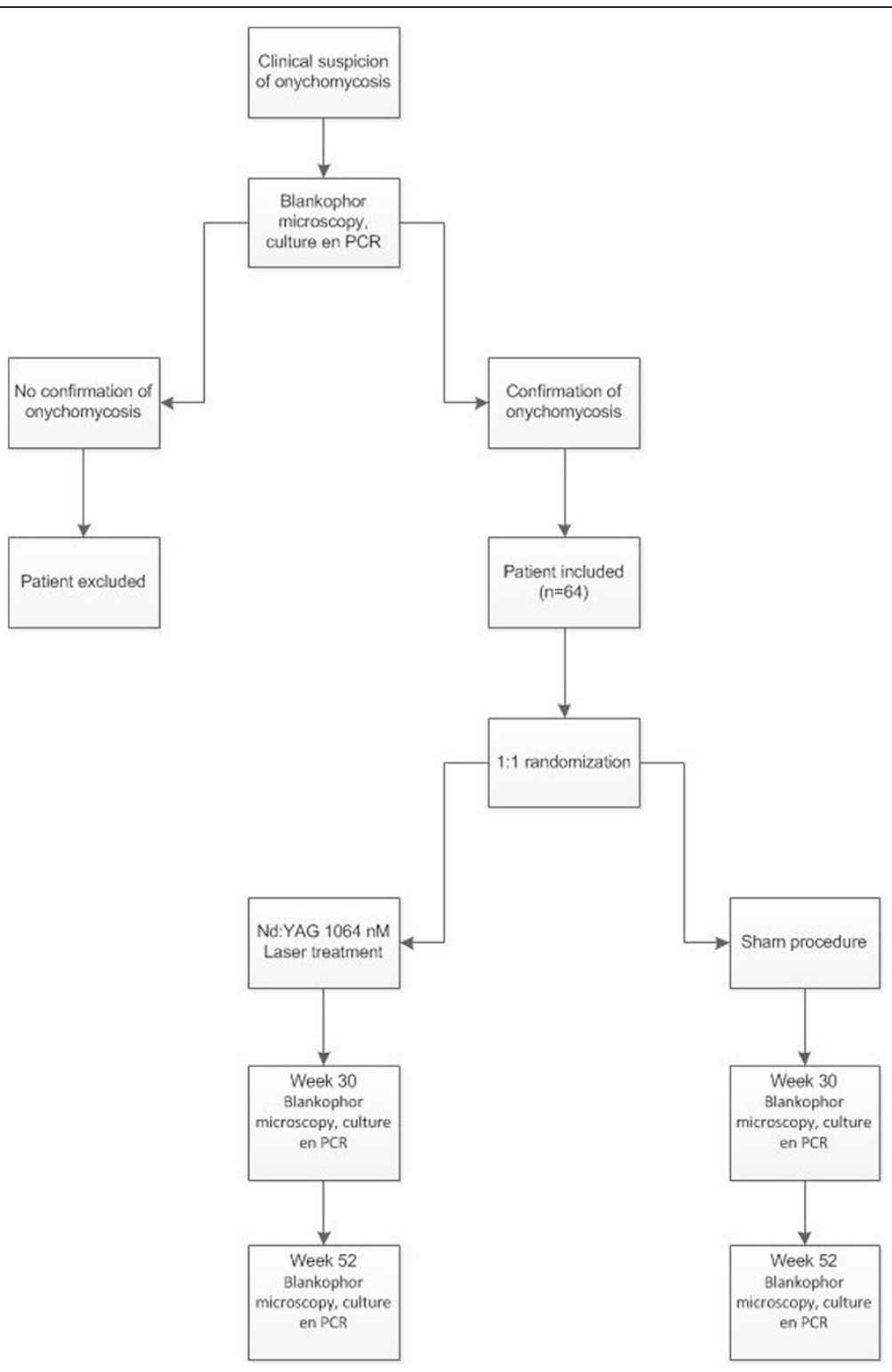

Figure 1 Study flow chart.

toe pressure below $50 \mathrm{mmHg}$, use of systemic or topical antifungal agents 3 months prior to inclusion, use of immunosuppressive drugs, presence of psoriasis, lichen planus, or other abnormalities that could result in clinically abnormal toenails, a history of epilepsy and insufficient knowledge of the Dutch language. Patients with a dark skin color (Fitzpatrick 4 and 5) are excluded since dark skin color is associated with dark nails, which theoretically lead to increased temperatures during laser application [33].

\section{Treatment assignment}

Patients will be randomized in blocks using sealed nontransparent envelopes to either laser or sham treatment. A cloth between the head and his or her feet and a blinded goggle (which also protects the eyes) is used for blinding the subjects. The periprocedural sounds and lights during the laser application and the sham procedure are identical. The investigators are blinded for treatment allocation through the use of an independent second podiatrist who performs the interventions. An independent three-person panel blinded for treatment allocation evaluates the study outcomes using standardized photographs made at baseline, week 30 and at the end of follow-up. When consensus cannot be reached, decisions are made through majority voting.

\section{Clinical follow-up}

Patients are treated with laser session in week 0, 2, 4, and 12. The settings are; $1064 \mathrm{~nm}$, spot size $3 \mathrm{~mm}, 20 \mathrm{~J} / \mathrm{cm} 2$, 
Table 1 The modified Simms' classification

\begin{tabular}{|c|c|c|}
\hline $\begin{array}{l}\text { Simm's } \\
\text { classification }\end{array}$ & Risk profile & Control frequency \\
\hline Simm's 0 & No loss of PS or PAV & Once in 12 months \\
\hline Simm's 1 & $\begin{array}{l}\text { Loss of PS or PAD, Without signs } \\
\text { of local increased pressure }\end{array}$ & Once in 6 months \\
\hline Simm's 2 & $\begin{array}{l}\text { Loss of PS and PAD. Loss of PS } \\
\text { and/or PAD in combination } \\
\text { with signs of local increased } \\
\text { pressure. }\end{array}$ & Once in 3 months \\
\hline Simm's 3 & $\begin{array}{l}\text { Ulcer or amputation in the } \\
\text { medical history }\end{array}$ & Once in 1 to 3 months \\
\hline
\end{tabular}

PAD, peripheral arterial disease; $\mathrm{PS}$, protective sensibility.

$5 \mathrm{~Hz}$, power $10 \mathrm{~W}$. A maximum of two sequential sessions (one session on the horizontal and one the vertical passing) will be applied to eliminate potential safety issues in those patients with a lack protective sensibility.

After 52 weeks, complete regrowth of the nail can be expected. At baseline, 30 and 52 weeks all target nails are sampled for blankophor microscopy, culture and polymerase chain reaction.

At baseline, 30 and 52 weeks, all nails are evaluated using the following scale: completely healed, markedly improved (less than 10\% affected), minor improvement, unchanged, and worse, and with the onychomycosis severity index [31]. The microbiological outcomes from week 30 are used to differentiate re-infection from persistent infection. Patients with re-infection have negative microbiological results at week 30 and positive results at week 52. Patients in whom different organisms at baseline and week 30 or 52 are isolated are regarded as having a reinfection. Patients are regarded as having a persistent infection when baseline, week 30 and week 52 microbiological results provide evidence of the same organism. Week 52 results, including the microbiological results, are used for evaluation of the primary and secondary outcomes. See Table 2 for the follow-up survey.

\section{Data analysis}

Estimating the proportion of patients with a complete cure rate to be $40 \%$ in the intervention group and $5 \%$ in the control group [27], with a power of 0.85 , an alpha of
0.05 (2-tailed), the sample size should be at least 56 . Accounting for loss to follow-up, the total sample size will be aimed at 64 .

Data entry is done in duplicate by persons not otherwise involved in the study. All statistical analyses are carried out by a statistician blinded for treatment allocation. The Fisher's Exact test will be used to test for differences between the treatment groups, and $95 \%$ confidence intervals (CIs) will be constructed for the differences in clinical and microbial cure rates. Normally distributed continuous variables will be compared using the Student $t$-test, and nonnormally distributed variables will be compared with the Mann-Whitney $U$ test. A significance level of less than 5\% is regarded as significant. Analyses will be performed according to the intention-to-treat principle. Separate perprotocol analyses are planned in patients in whom all treatment sessions are performed and for analyses excluding re-infection. A sensitivity analysis is planned that regards completely cured patients at week 30 who have a new infection at week 52 as having a complete cure. All endpoints are evaluated at week 52 .

\section{Discussion}

Patients with diabetes who suffer from onychomycosis and neuropathy or PAD have an increased risk for developing diabetes-related foot complications, but also for treatment-related complications. New, effective and safe treatment modalities are needed for patients with diabetes who have risk factors for diabetes-related foot complications, in order to minimize the risks of developing ulcers and also to minimize discomfort and treatment-related complications caused by the current usual care.

The results of this study will provide the first doubleblinded evidence for laser therapy and the first study in patients with diabetes mellitus with risk factors for developing diabetic foot ulcers. Positive results could aid in the prevention of diabetes-related foot complications since no local curative options are available, and systemic treatment could be avoided. If the results were to show safety and efficacy of laser therapy, this would provide the first high-level evidence for efficacy of a local

Table 2 Follow-up survey

\begin{tabular}{|c|c|c|c|c|c|c|c|}
\hline & Baseline & Week 0 & Week 2 & Week 4 & Week 12 & Week 30 & Week 52 \\
\hline & & Session 1 & Session 2 & Session 3 & Session 4 & & \\
\hline Inclusion & & $x$ & & & & & \\
\hline Randomization & & $x$ & & & & & \\
\hline Blankophor microscopy/Culture & $x$ & & & & & $x$ & $x$ \\
\hline \multicolumn{8}{|l|}{$/ P C R$} \\
\hline Photo & $x$ & & & & $x$ & $x$ & $x$ \\
\hline Treatment & & $x$ & $x$ & $x$ & $x$ & & \\
\hline
\end{tabular}


treatment modality against onychomycosis. The results of this study will be at least equally relevant if they were to show non-efficacy since the use of lasers is becoming increasingly popular. In that case, unnecessary laser applications and possible harm could be avoided.

\section{Trial status}

\section{Recruiting.}

\section{Abbreviations}

Cl: confidence intervals; FDA: Food and Drug Administration; HRQOL: healthrelated quality of life; OSI: Onychomycosis Severity Index; PAD: peripheral arterial disease; PCR: polymerase chain reaction; QoL: quality of life.

\section{Competing interests}

One of the employers of $L$. Nijenhuis has a stake in a company that supplies medical devices, among others, the laser used for this study, L. Nijenhuis has no stake in this company. All other authors declare that they have no competing interests.

\section{Authors' contributions}

GL and NK conceived the study. GL, LN and NK developed the study protocol. HB, MW, KG made critical revisions to the manuscript. MW supervises the microbiological study. KG performed the statistical planning. All authors have reviewed and approved the final version of the manuscript.

\section{Acknowledgements}

The Hein Hogerzeil foundation funded starting costs and microbiological costs in the form of a grant. The Dutch Diabetes Research Foundation has awarded a grant for this study (grant nr. 2014.00.1741).

\section{Author details}

'Diabetes Centre, Isala, Dr Spanjaardweg 11, 8025 BT Zwolle, the Netherlands. ${ }^{2}$ Innofeet Voetencentrum Nijenhuis Podiatry, Simon Stevinweg 13, 8013 NA Zwolle, the Netherlands. ${ }^{3}$ Department of Internal Medicine, University of Groningen, University Medical Center Groningen, Hanzeplein 1, 9700 RB Groningen, the Netherlands. "Langerhans Medical Research Group, Postbus 21, 4254 ZG Sleeuwijk, the Netherlands. ${ }^{5}$ Department of Medical Microbiology, Isala, Dr. van Heesweg 28025 AB Zwolle, the Netherlands. ${ }^{6}$ Department of General Practice, University of Groningen, University Medical Center Groningen, Hanzeplein 1, 9700 RB Groningen, the Netherlands. ${ }^{7}$ Department of Internal Medicine, Gelre Hospital, Albert Schweitzerlaan 31, 7334 DZ Apeldoorn, the Netherlands.

\section{Received: 27 November 2014 Accepted: 27 February 2015}

Published online: 22 March 2015

\section{References}

1. Williams R, Van Gaal L, Lucioni C. Assessing the impact of complications on the costs of Type II diabetes. Diabetologia. 2002;45:S13-7.

2. Boulton AJ, Vinik Al, Arezzo JC, Bril V, Feldman EL, Freeman R, et al. Diabetic neuropathies: a statement by the American Diabetes Association. Diabetes Care. 2005;28:956-62

3. Gupta A, Konnikov N, MacDonald P, Rich P, Rodger N, Edmonds M, et al. Prevalence and epidemiology of toenail onychomycosis in diabetic subjects: a multicenter study. Br J Dermatol. 1998;139:665-71.

4. Boyko E, Ahroni J, Cohen V, Nelson K, Heagerty P. Prediction of diabetic foot ulcer occurrence using commonly available clinical information: the seattle Diabetic Foot Study. Diabetes Care. 2006;29:1202-7.

5. Cathcart S, Cantrell W, Elewski B. Onychomycosis and diabetes. J Eur Acad Dermatol Venereol. 2009;23:1119-22.

6. Rich P. Onychomycosis and tinea pedis in patients with diabetes. J Am Acad Dermatol. 2000:43:130-8.

7. Armstrong D, Holtz K, Wu S. Can the use of a topical antifungal nail lacquer reduce risk for diabetic foot ulceration? results from a randomised controlled pilot study. Int Wound J. 2005;2:166-70.

8. Brodell J, Brodell R. Recurrent lymphangitic ellulitis syndrome. Contemp Orthop. 1992;25:461-8.
9. Cox N, Colver G, Paterson W. Management and morbidity of cellulitis of the leg. J R Soc Med. 1998;91:634-7.

10. Pierce R, Daugird A. Recurrent leg cellulitis: pathogenesis, prevention and treatment. J Am Board Fam Pract. 1992;5:85-7.

11. Armstrong D. Is diabetic foot care efficacious or cost-effective? Ostomy Wound Manage. 2001:47:28-32.

12. Bild D, Shelby J, Sinnock P, Browner W, Braveman P, Showstock J. Lower extremity amputations in people with diabetes: epidemiology and prevention. Diabetes Care. 1989;12:24-9.

13. Block P. The diabetic foot ulcer: a complex problem with a simple treatment approach. Mil Med. 1981;146:644-6.

14. Lavery L, Armstrong D, Vela S, Quebedeaux T, Fleischli J. Practical criteria for screening patients at high risk for diabetic foot ulceration. Arch Intern Med. 1998;158:158-62.

15. Smith D, Weinberger $M$, Katz B. A controlled trial to increase office visits and reduce hospitalization in diabetic patients. J Gen Intern Med. 1987;2:232-8.

16. Gupta A, Humke S. The prevalence and management of onychomycosis in diabetic patients. Eur J Dermatol. 2000;10:379-84.

17. Karchmer A, Gibbons G. Foot infection in diabetes: evaluation and management. Curr Clin Top Infect Dis. 1994;14:1-22.

18. Ramsey S, Newton K, Blough D, McCulloch D, Sandhu N, Reiber G, et al. Incidence, outcomes, and cost of foot ulcers in patients with diabetes. Diabetes Care. 1999;22:383-7

19. Shuster S, Fisher GH, Harris E, Binnell D. The effect of skin disease on self image [proceedings]. Br J Dermatol. 1978;99:18-9.

20. Shuster S. Depression of self-image by skin disease. Acta Derm Venereol Suppl (Stockh). 1991;156:53.

21. Drake LA, Scher RK, Smith EB, Faich GA, Smith SL, Hong JJ, et al. Effect of onychomycosis on quality of life. J Am Acad Dermatol. 1998;38:702-4.

22. Drake LA, Patrick DL, Fleckman P, Andr J, Baran R, Haneke E, et al. The impact of onychomycosis on quality of life: development of an international onychomycosis-specific questionnaire to measure patient quality of life. $J$ Am Acad Dermatol. 1999:41:189-96.

23. Warshaw EM, Foster JK, Cham PM, Grill JP, Chen SC. NailQoL: a quality-of-life instrument for onychomycosis. Int J Dermatol. 2007:46:1279-86.

24. Robbins J. Treatment of onychomycosis in the diabetic population. $J$ Diabetes Complications. 2003:17:98-104.

25. Apelqvist J, Larrson J. What is the most effective way to reduce incidence of amputation in the diabetic foot? Diabetes Metab Res Rev. 2000;16:575-83.

26. Singh N, Armstrong D, Lipsky B. Preventing foot ulcers in patients with diabetes. J Am Med Assoc. 2005;293:217-28.

27. Gupta AK, Simpson FC. New therapeutic options for onychomycosis. Expert Opin Pharmacother. 2012;13:1131-42.

28. Anarella J, Toth C, Debello J. Preventing complications in the diabetic patient with toenail onychomycosis. J Am Podiatr Med Assoc. 2001;91:325-8.

29. Bhatta AK, Huang X, Keyal U, Zhao JJ. Laser treatment for onychomycosis: a review. Mycoses. 2014;57:734-40.

30. Arabatzis M, van Coppenraet LEB, Kuijper EJ, de Hoog GS, Lavrijsen AP, Templeton K, et al. Diagnosis of common dermatophyte infections by a novel multiplex real-time polymerase chain reaction detection/identification scheme. Br J Dermatol. 2007;157:681.

31. Carney C, Tosti A, Daniel R, Scher R, Rich P, DeCoster J, et al. A new classification system for grading the severity of onychomycosis: Onychomycosis Severity Index. Arch Dermatol. 2011;147:1277-82.

32. Nederlandse Internisten Vereeniging. Richtlijn Diabetische voet. Van Zuiden Communications B.V., Alphen aan den Rijn, ISBN-10 90-8523-142-6, 2007.

33. Rao K, Sankar TK. Long-pulsed Nd:YAG laser-assisted hair removal in Fitzpatrick skin types IV-VI. Lasers Med Sci. 2011;26:623-6. 